$$
\text { "bair" — 2008/4/9 — 9:42 — page 377 — \#1 }
$$

\title{
From Newton's Fluxions to Virtual Microscopes
}

\author{
JACQUes BAIR and VALerie Henry
}

\begin{abstract}
The method of fluxions was originally given by Newton among others in order to determine the tangent to a curve. In this note, we will formulate this method by the light of some modern mathematical tools: using the concept of limit, but also with hyperreal numbers and their standard parts and with dual numbers; another way is the use of virtual microscopes both in the contexts of classical and non standard analysis.
\end{abstract}

Key words and phrases: fluxions, tangent lines, hyperreal numbers, dual numbers, virtual microscopes, non standard analysis.

ZDM Subject Classification: C34, D54, I44.

In his Latin work "Methodus Fluxiorum", which was originally written in 1671, sir Isaac Newton studied planar curves with a mechanical point of view: he considered time, which was an implicit variable, as increasing by continual flux, while the two current variables $x$ and $y$ are increasing continually in time; for example, the equation

$$
x^{2}+y^{2}=1
$$

determines the curve which is described by a point moving along a circle with an unitary radius.

For Newton [11], the increasing of the variable $x$ (resp. $y$ ) during an infinitely small period of time is proportional to its fluxion $\dot{x}$ (resp. $\dot{y}$ ), i.e. the speed with which $x$ (resp. $y$ ) increases: more precisely, such a variation of $x$ (resp. $y$ ) is equal to $\dot{x} o$ (resp. $\dot{y} o$ ), where o denotes an infinitely small quantity of time.

Copyright (c) 2007 by University of Debrecen
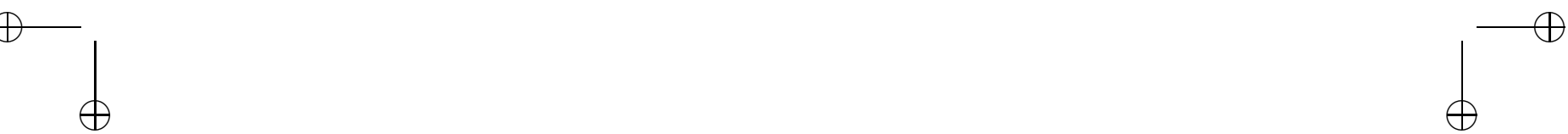


$$
\text { "bair" — 2008/4/9 — 9:42 — page 378 — \#2 }
$$

Newton applied this principle to solve problems about tangents to an algebraic curve $\mathcal{C}$ with equation

$$
f(x, y)=0,
$$

$f$ being a polynomial function with two real variables $x$ and $y$ and defined by

$$
f(x, y)=\sum_{j=0}^{m} \sum_{k=0}^{p} a_{j k} x^{j} y^{k}
$$

where all the coefficients $a_{j k}$ are real while $m$ and $p$ are natural integers. Let $P=(r, s)$ be a point of $\mathcal{C}$ : we have

$$
f(r, s)=0
$$

whereas $r$ (resp. $s$ ) is the position reached by the variable $x$ (resp. $y$ ) at a time $t_{0}$. During the interval of time $\left[t_{0}, t_{0}+\Delta t\right]$, the point moves from $P$ to $P_{1}=\left(r_{1}, s_{1}\right)$ with an uniform motion: if $\dot{x}$ (resp. $\dot{y}$ ) denotes the constant speed of $x$ (resp. $y$ ), we have

$$
r_{1}=r+\dot{x} \Delta t \text { and } s_{1}=s+\dot{y} \Delta t .
$$

If $\Delta t=o$ is infinitely small, then the point $P_{1}$ also lies on $\mathcal{C}$ and thus

$$
f(r+\dot{x} o, s+\dot{y} o)=0 .
$$

Because $o$ is infinitely small, the powers of $\dot{x} o$ and of $\dot{y} o$ with an exponent greater than 1 can be neglected; this leads to a simple equation which gives the value of $\frac{\dot{y}}{\dot{x}}$ : it is the slope of the tangent to the curve $\mathcal{C}$ at point $P$ (see Figure 1 ).

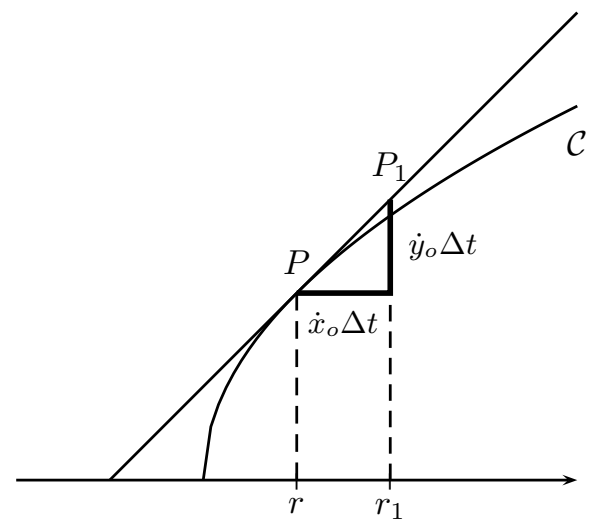

Figure 1. Curve $\mathcal{C}$ and its tangent at $P$ 


$$
\text { "bair" — 2008/4/9 — 9:42 — page 379 — \#3 }
$$

As an illustration, consider the curve $\mathcal{C}$ whose equation is

$$
f(x, y)=x^{3}+x-3 x^{2} y+y^{2}=0
$$

and a point $P=(r, s)$ on $\mathcal{C}$. This last equality is preserved by putting

$$
r+\dot{x} o \text { and } s+\dot{y} o
$$

respectively instead of $r$ and $s$. So, we have:

$$
(r+\dot{x} o)^{3}+(r+\dot{x} o)-3(r+\dot{x} o)^{2}(s+\dot{y} o)+(s+\dot{y} o)^{2}=0
$$

whence, because $f(r, s)=0$ :

$$
\begin{aligned}
\left(\left(3 r^{2}-6 r s+1\right) \dot{x}+\right. & \left.\left(2 s-3 r^{2}\right) \dot{y}\right) o+ \\
& \left(3 r-6 r \dot{x} \dot{y}-3 s \dot{x}^{2}+\dot{y}^{2}\right) o^{2}+\left(\dot{x}^{3}-3 \dot{x}^{2} y\right) o^{3}=0
\end{aligned}
$$

If the terms with $o^{2}$ and $o^{3}$ are suppressed, then one gets after dividing by $o$

$$
\left(3 r^{2}-6 r s+1\right) \dot{x}+\left(2 s-3 r^{2}\right) \dot{y}=0
$$

whence, if $2 s \neq 3 r^{2}$

$$
\frac{\dot{y}}{\dot{x}}=\frac{3 r^{2}-6 r s+1}{3 r^{2}-2 s}
$$

this last result is the slope of the tangent to $\mathcal{C}$ at $P$ and coincides, following the rule of implicit differentiation, with

$$
-\frac{\frac{\partial f}{\partial x}(r, s)}{\frac{\partial f}{\partial y}(r, s)}
$$

For the successors of Newton, this reasoning had two disadvantages. First, it uses infinitesimals, i.e. infinitely small numbers, whose existence could not be proved and raised tremendous objections. Secondly, some terms, like $(o \dot{x})^{2}$, are suppressed whereas they can not be equal to 0 because it is later on necessary to divide by $o$.

There exist a few modern and rigorous explanations for the Newton's method; we shall present them on our example.

A classical way consists to consider the equalities

$$
x=r+\dot{x} \Delta t+\varepsilon_{1}(\Delta t) \text { and } y=s+\dot{y} \Delta t+\varepsilon_{2}(\Delta t)
$$




$$
\text { "bair" — 2008/4/9 — 9:42 — page 380 — \#4 }
$$

when

$$
\lim _{\Delta t \rightarrow 0} \frac{\varepsilon_{i}(\Delta t)}{\Delta t}=0 \text { for } i=1,2 .
$$

By easy computations similar to the above ones on the two members of (1), we get the desired solution by taking the limit for $\Delta t \rightarrow 0$.

As another way, we work in the context of the non standard analysis, first developed by Robinson who has constructed the ordered field of the hyperreal numbers [10]: it is an extension of the real field $\mathbb{R}$ where there especially exist positive infinitely small numbers, i.e. hyperreal numbers which are positive but less than every positive real number. In 1961, thanks to the recent developments of the mathematical logic, Robinson was able to prove the existence of such infinitely small numbers and to justify the results found in the end of the XVII ${ }^{\text {th }}$ century.

So, we can use the Newton's formulas (2) where $o$ denotes an infinitely small number, we also obtain the equality (4) and the conclusion is found intuitively by taking the real observable value of each hyperreal number: formally, it consists to take the standard parts [8] of the considered hyperreal numbers.

A more surprising way, developed in [5], consists to work with dual numbers introduced by Clifford [4]: they are "a particular two-dimensional commutative unital associative algebra over the real numbers, arising from the reals by adjoining one new element $\varepsilon$ with the property $\varepsilon^{2}=0$ ". Indeed, if the expression $o$ is taken equal to this $\varepsilon$ dual number in the Newton's reasoning over the example (1), then the desired result is immediate.

In the following, we propose a modern approach of the Newton's method, by using the concept of virtual microscope $([3],[6],[7],[8])$. The use of this tool by mathematicians has recently spread with the coming of non standard analysis. However it is not new, as proved by this citation of Pascal in 1654, as he wanted to give an advise to those who had difficulties to conceive infinitely small quantities

s'ils ne peuvent comprendre que des parties si petites, qu'elles nous sont imperceptibles, puissent être autant divisées que le firmament, il n'y a pas de meilleur remède que de les leur faire regarder avec des lunettes qui grossissent cette pointe délicate jusqu'à une prodigieuse masse ${ }^{1}$ ([9, p. 30]).

1. if they can't understand that so small quantities, imperceptible for human being, can be as much divided as the firmament, no solution is better than looking at these quantities through glasses which enlarge this delicate point into a phenomenal mass. 


$$
\text { "bair" — 2008/4/9 — 9:42 — page 381 — \#5 }
$$

The goal is here to have a look on the curve $\mathcal{C}$ in a very small neighbourhood of the considered point $P$. So, it seems natural to try to "enlarge" the graphical representation of the curve around $P$; for that, a virtual microscope is an adequate tool.

As an illustration in the plane, let us guide on point $P=(1,1)$ a "microscope" which enlarges all the lengths by a factor 10: $P$ is seen in the "center" of the ocular, i.e. at the intersection of two new horizontal and vertical axes, and, for example, the square $[0.9,1.1] \times[0.9,1.1]$ becomes equal to the new square $[-1,1] \times$ $[-1,1]$ (see Figure 2).

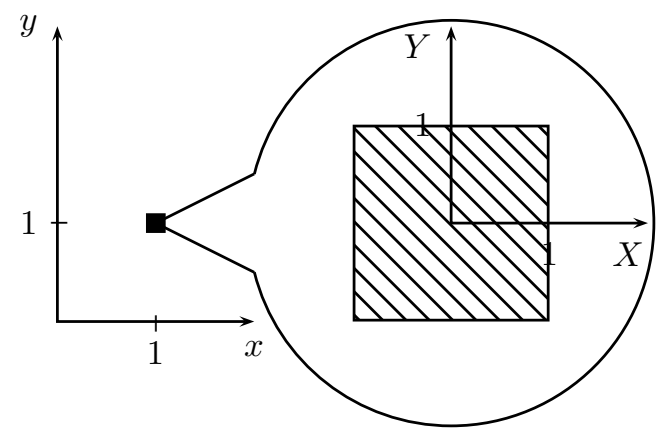

Figure 2. Illustration of the use of a microscope

Mathematically, such a virtual microscope leads to a transformation in the plane $\mathbb{R}^{2}$ defined by

$$
(x, y) \mapsto(10(x-1), 10(y-1)) .
$$

More generally, we consider a virtual microscope directed to point $P=(r, s)$ and which enlarges $n$ times all the vertical and horizontal lengths; so, we consider the mapping, from $\mathbb{R}^{2}$ to $\mathbb{R}^{2}$, given by

$$
(x, y) \mapsto(X, Y)=(n(x-r), n(y-s)) ;
$$

the new coordinates $X$ and $Y$ are such that

$$
x=r+\frac{X}{n} \text { and } y=s+\frac{Y}{n}
$$

If we apply this last transformation to equation (1), we get

$$
f\left(r+\frac{X}{n}, s+\frac{Y}{n}\right)=0
$$




$$
\text { "bair" — 2008/4/9 — 9:42 — page 382 — \#6 }
$$

and so, we obtain equality (4) in which

$$
\dot{x} o=\frac{X}{n} \text { and } \dot{y} o=\frac{Y}{n} ;
$$

so, we have the image of the curve $\mathcal{C}$ in the ocular of our microscope. The conclusion is obtained by taking a limit for $n \rightarrow+\infty$ (see [2]).

It is possible to see geometrically what happens with this method. Indeed, if we take, for example, the point $P=(1,1)$, we can plot the curve $\mathcal{C}$ first in the neighbourhood $[0,2] \times[0,2]$ of $P$ and then we divide each length respectively by factors $n=2,10,100$ : this corresponds to the use of microscopes pointed on $P$ which enlarge 2,10, 100 times; progressively, the curve becomes nearer and nearer to a straight line (see Figures 3).
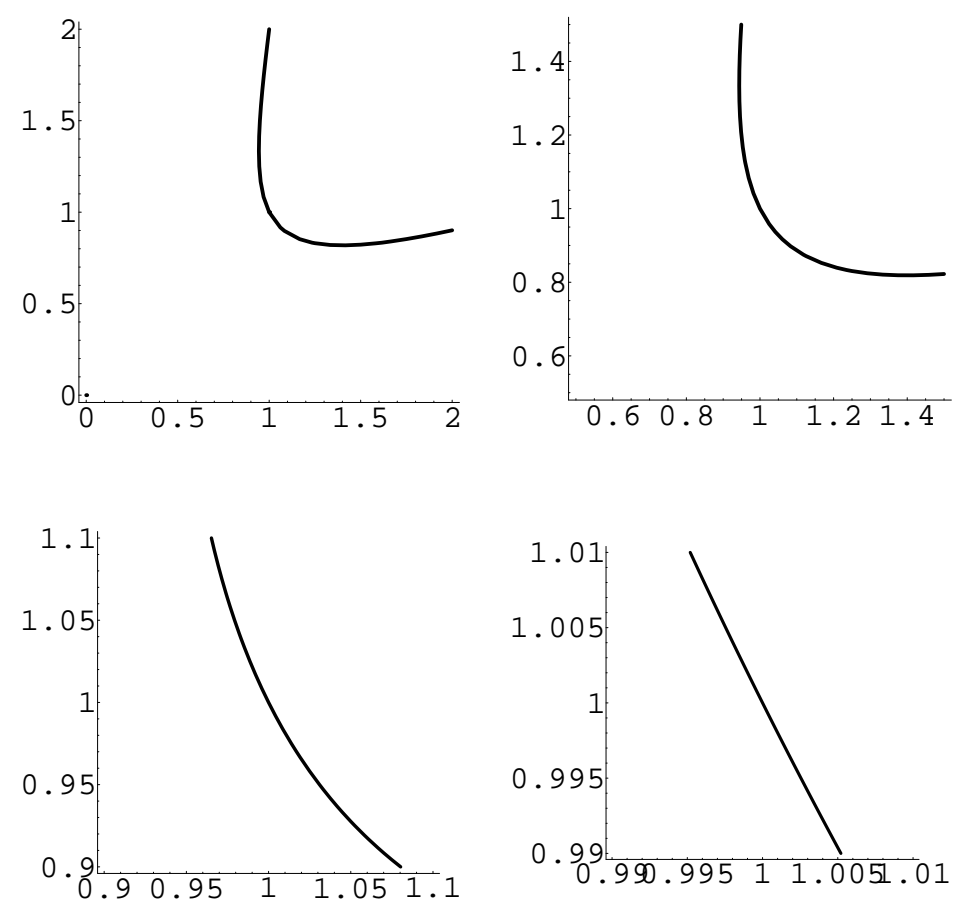

Figure 3. Geometric illustration

Clearly, the curve drawn on the last figure is similar to the tangent of $\mathcal{C}$ at $P$.

It is also possible to work in the context of hyperreal numbers by using a sole virtual microscope directed to $P$ and which enlarges $\omega$ times every length, where 


$$
\text { "bair" — 2008/4/9 — 9:42 — page 383 — \#7 }
$$

$\omega$ is an infinite number, i.e. a hyperreal number greater than every real number [8]. Then, the conclusion holds by taking the standard parts of the two members of equality (4) in which

$$
\dot{x} o=\frac{X}{\omega} \text { and } \dot{y} o=\frac{Y}{\omega}
$$

In conclusion, Newton's method of fluxions can be rigorously justified by a few modern and various arguments, some of them being intuitive and other more abstract. In consequence, this works shows a subsequent development of the concepts used by mathematicians. Especially, the context of the hyperreal numbers is very close to the Newton's ideas and seems convenient for solving local problems in mathematical analysis. This confirms a recent thought about the fact that non standard analysis deserves a place among the fundamental methods to be used by mathematicians of future generations [1].

\section{References}

[1] L. Arkeryd, Nonstandard Analysis, American Mathematical Monthly 112 (2005), 926-928.

[2] A. Antibi, J. Bair et V. Henry, Limites de courbes : théorie et applications en analyse, Mathématiques et Pédagogie 147 (2004), 65-87.

[3] A. Antibi, J. Bair et V. Henry, Une modélisation d'un zoom au moyen de microscopes virtuels, Teaching Mathematics and Computer Science 2, no. 2 (2004), 319-335.

[4] W. K. Clifford, Preliminary Sketch of Biquaternions, Proc. London Mathematical Society 4 (1873), 361-395.

[5] R. Godement, Analyse mathématique I : convergence, fonctions élémentaires, Springer-Verlag, Berlin Heidelberg, 1998.

[6] V. Henry, Nouvelle approche pour introduire la notion de tangente à une courbe, Mathématiques et Pédagogie 145 (2004), 65-79.

[7] V. Henry, Tangentes aux coniques : méthodes passées, présentes et à venir, Mathématiques et Pédagogie 152 (2005), 41-75.

[8] H. J. Keisler, Elementary Calculus, Prindle, Weber \& Schmidt Inc., Boston, 1976.

[9] B. Pascal, L'esprit de la Géométrie - De l'art de persuader, Univers des Lettres, Bordas, Paris, 1986.

[10] A. Robinson, Non-Standard Analysis, Koninkl. Ned. Akad. Wetensch. Proc., Serie A, 64 (1961), 432-440.

[11] D. T. Whiteside, The Mathematical Papers of Isaac Newton III, Cambridge University Press, 1969. 
JACQUES BAIR and VALERIE HENRY

HEC-MANAGEMENT SCHOOL

UNIVERSITY OF LIEGE

BAT B31

BOULEVARD DU RECTORAT, 7

4000 LIEGE

BELGIUM

E-mail: J.Bair@ulg.ac.be

E-mail: V.Henry@ulg.ac.be

(Received July, 2007) 